\title{
Angiosarcoma esplénico primario con rotura capsular y diseminado: reporte de un caso
}

\author{
Primary splenic angiosarcoma with capsular rupture and disseminated: a case report
}

Javier A. Teco-Cortes ${ }^{*}$, Juan J. Navarrete-Pérez y Óscar E. Sánchez-Castro ${ }^{2}$

${ }^{1}$ Departamento de Patología, Hospital General de México Eduardo Liceaga; ${ }^{2}$ Departamento de Patología, Centro Médico Naval. Ciudad de México, México

\begin{abstract}
Resumen
El angiosarcoma esplénico primario es una neoplasia vascular maligna con un pronóstico mortal a corto plazo en la mayoría de los casos, que se presenta con síntomas inespecíficos y usualmente en estadios avanzados. Presentamos el caso de un hombre de 49 años, con historia de diarrea crónica y pérdida de peso, en el que se identificó esplenomegalia. El estudio patológico reveló el diagnóstico de angiosarcoma primario de bazo, con diseminación en hígado, pulmón y ganglios linfáticos. Debido a la rareza de esta neoplasia existe poca experiencia en relación con el mejor manejo con impacto significativo en la supervivencia de los pacientes.
\end{abstract}

Palabras clave: Angiosarcoma. Bazo. Esplenomegalia.

\begin{abstract}
Primary splenic angiosarcoma is a malignant vascular neoplasm with a short-term fatal prognosis in most cases, with nonspecific symptoms and usually in advanced stages. We present the case of a 49-year-old man with clinical history of chronic diarrhea and weight loss, in which it was identifies splenomegaly. The histopathological study disclose the diagnosis of primary angiosarcoma of the spleen, with spread to the liver, lung, and lymph nodes. The patient was discharged after undergoing splenectomy, with palliative treatment. Because the rarity of this neoplasm, there is limited experience regarding the best management with a significant impact on patient survival.
\end{abstract}

Key words: Angiosarcoma. Spleen. Splenomegaly.

\section{Introducción}

El angiosarcoma primario de bazo es una neoplasia maligna de origen vascular, poco frecuente y con un comportamiento biológico muy agresivo. Suele presentarse en pacientes por arriba de 40 años, sin una predilección por sexo significativa. Su presentación clínica, así como los hallazgos por estudios de laboratorio e imagen, son inespecíficos; entre ellos pueden destacarse dolor abdominal superior, anemia y aumento en el tamaño del bazo. El diagnóstico definitivo se realiza mediante análisis histopatológico.

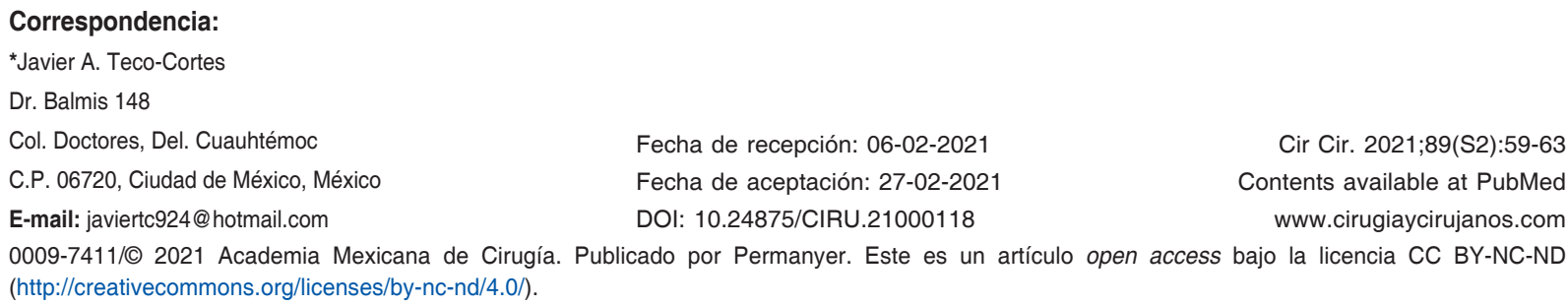


Suele presentarse con afección de otros órganos, como hígado, pulmón, ganglios linfáticos y hueso. Del $25 \%$ al $69 \%$ de los casos se presentan con rotura esplénica. El tratamiento de primera opción es la esplenectomía, pero su pronóstico es casi siempre fatal a los pocos meses del diagnóstico $0^{1-3}$.

\section{Caso clínico}

Varón de 49 años, con peso habitual de $102.2 \mathrm{~kg}$, que como únicos antecedentes de importancia presenta diabetes mellitus tipo 2 de 20 años e hipertensión arterial sistémica de 10 años de evolución, en tratamiento médico. Inició su padecimiento 3 meses previos a su hospitalización, con diarrea acuosa persistente y pérdida de $18.0 \mathrm{~kg}$ en 2 meses. Ingresó por cuadro de dolor abdominal referido desde una clínica privada con reporte ultrasonográfico abdominal y tomografía computarizada de abdomen que mostraba esplenomegalia de $26.9 \times 14.0 \times 21.9 \mathrm{~cm}$, parénquima homogéneo y escaso líquido periesplénico, con sospecha de linfoma de la zona marginal esplénica, así como reporte de biopsia de médula ósea con mielofibrosis de grado I. En la exploración física se identificó un abdomen globoso a expensas de tumor abdominal de consistencia pétrea, que sobresalía $2.0 \mathrm{~cm}$ por debajo del reborde costal izquierdo hasta la cicatriz umbilical, y dolor abdominal superficial y profundo, difuso y de gran intensidad, con síndrome de abdomen agudo. En los exámenes de laboratorio se identificaron leucocitosis $(13.8 \times$ $\left.10^{3} / \mu \mathrm{l}\right)$, anemia normocítica normocrómica $(8.4 \mathrm{~g} / \mathrm{dl})$, trombocitopenia $\left(59 \times 10^{3} / \mu \mathrm{l}\right)$ e hiperglucemia $(182 \mathrm{mg} / \mathrm{dl})$; el resto de los parámetros estaban dentro de la normalidad. Se realizó laparotomía exploradora y se identificaron esplenomegalia y múltiples adherencias del bazo con el estómago, el páncreas, el colon y el diafragma, así como múltiples lesiones nodulares en la superficie del hígado, por lo que se realizaron esplenectomía y omentectomía, y se tomó biopsia en cuña del hígado.

El bazo midió $31.0 \times 25.0 \times 10.0 \mathrm{~cm}$ y pesó $5200 \mathrm{~g}$. La superficie externa era de color rojo violáceo, lisa y renitente, con múltiples soluciones de continuidad; al corte, la superficie era heterogénea, de color rojo violáceo, aterciopelada, entremezclada con zonas de color blanco-gris mal delimitadas (Fig. 1). Microscópicamente se identificó necrosis en el $90 \%$ del tejido con áreas de hemorragia entremezclada, y en las áreas de tejido viable se identificó una lesión neoplásica heterogénea, compuesta sobre todo por grandes trayectos
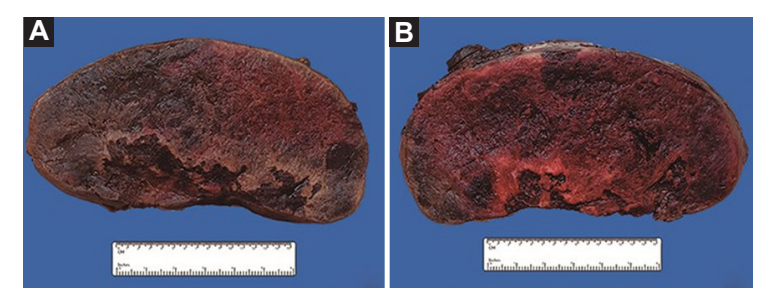

Figura 1. Angiosarcoma esplénico. A: superficie de corte del bazo, fijada en formol, en la que se identifica una lesión heterogénea que sustituye gran parte de la superficie, mal delimitada, de color café rojizo, con aspecto aterciopelado y consistencia blanda. Además, se identifican áreas extensas de aspecto congestivo-hemorrágico y necrosis. B: superficie de corte del bazo, en fresco, en la que se identifica con mayor detalle el aspecto predominantemente sólido aterciopelado con áreas microquísticas, así como el aspecto congestivo-hemorrágico de la neoplasia.

vasculares irregulares recubiertos de células endoteliales con atipia moderada a pleomórficas, así como áreas focales sólidas con formación de pequeños trayectos vasculares dentro de un estroma hialino. Otros campos mostraron estructuras papilares dentro de los trayectos vasculares. Se identificó una mitosis por cada $10 \mathrm{cam}$ pos a gran aumento y hematopoyesis extramedular (Fig. 2). Las células neoplásicas fueron positivas para CD31, CD34, factor VIII, trombomodulina y vimentina, y focalmente para actina de músculo liso, con Ki-67 mayor del 30\%, y negativas para CKAE1/AE3, calretinina y HMB-45 (Fig. 3) por inmunohistoquímica. Se estableció el diagnóstico de angiosarcoma primario de bazo (patrón convencional $80 \%$, sólido $10 \%$ y papilar $10 \%)$. En la biopsia hepática se encontraron metástasis. En el epiplón se identificó peritonitis aguda y crónica fibrinosa.

El paciente fue dado de alta 5 días después de la cirugía, peró presentó datos de neumonía nosocomial y fue nuevamente hospitalizado. Fue tratado con antibióticos y se realizó tomografía computarizada abdominal de control, en la que se identificaron un absceso subfrénico izquierdo (32.4 ml), hepatomegalia con múltiples lesiones nodulares en el hígado y las superficies pleurales, y aumento de tamaño de los ganglios linfáticos cervicales, mediastinales y axilares, sugerentes de un proceso metastásico. Ante la evidencia de un proceso neoplásico diseminado, se optó por dar manejo paliativo, por lo que fue dado de alta. El paciente fue perdido al seguimiento.

\section{Discusión}

El angiosarcoma esplénico primario es una neoplasia maligna de origen vascular que fue descrita por 

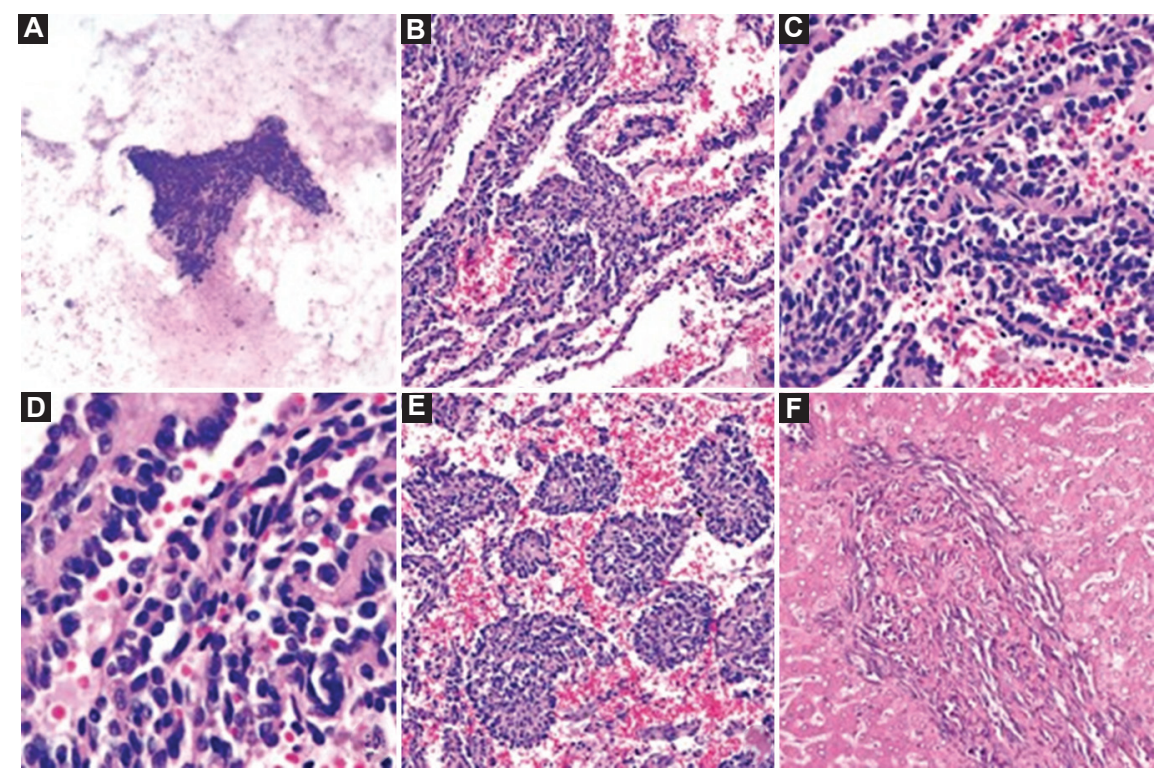

Figura 2. Angiosarcoma esplénico. A: extendido citológico en el que se identifican células neoplásicas fusiformes con atipia marcada, cohesivas y moderado citoplasma, rodeadas de eritrocitos (hematoxilina y eosina $50 x$ ). B: múltiples canales vasculares grandes e irregulares recubiertos por células endoteliales con atipia marcada, y abundantes eritrocitos en las luces (hematoxilina y eosina 50 x). C y D: vista a mayor aumento de las áreas vasoformativas en las que se aprecia con mayor detalle la atipia de las células endoteliales (hematoxilina y eosina 100 y 200 x). E: se identificaron áreas con patrón papilar de forma focal (hematoxilina y eosina 50 x). F: lesión metastásica con formación de pequeños canales vasculares en el hígado $(50 \times)$.

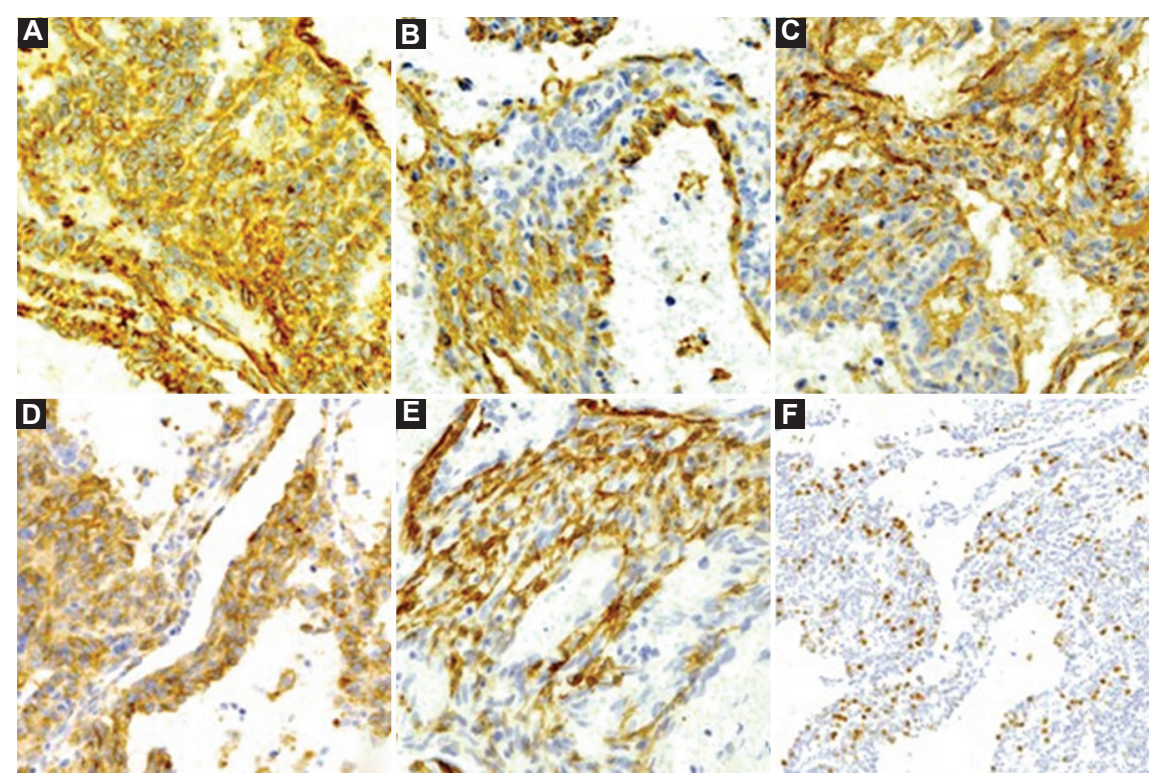

Figura 3. Angiosarcoma esplénico. Las reacciones de inmunohistoquímica fueron positivas para: A: CD31. B: CD34. C: factor VIII. D: trombomodulina. E: focalmente para actina de músculo liso (en el 10\% de las células neoplásicas) y vimentina (no mostrada); y negativas para CKAE1/ AE3, HMB45 y calretinina (no mostradas); F: El índice de proliferación es mayor del 30\% definido por Ki-67.

primera vez en 1879 por Theodor Langhans ${ }^{4}$, y desde entonces se han publicado pocos casos en la literatura.

El rango de edad de los pacientes reportados es amplio, desde la infancia hasta la vejez, pero en su mayoría son mayores de 40 años, con un promedio de 59 años; los hombres a edades más tempranas (53 años en promedio) que las mujeres (68 años en promedio). A aunque se ha reportado un ligero predominio en hombres, no hay clara predilección por un 
sexo $^{1,2,5}$. Nuestro caso es un hombre de 49 años, una edad por debajo del promedio general, incluso para el sexo masculino.

La etiología de los angiosarcomas esplénicos es desconocida, aunque se han reportado casos asociados con antecedente de quimioterapia o neoplasias metacrónicas, como linfoma no Hodgkin, cáncer de mama y carcinoma papilar de tiroides. Estos casos son aislados y no está establecida una asociación causal ${ }^{1,2,6}$. En nuestro paciente, los únicos antecedentes de relevancia fueron los crónico-degenerativos, sin causalidad inmediata.

Clínicamente, la sintomatología es inespecífica. Con mayor frecuencia se presentan dolor abdominal superior, pérdida de peso y tumor abdominal en el cuadrante superior izquierdo. El $25-59 \%$ se presentan con rotura esplénica asociada al aumento masivo del tamaño del bazo. Los hallazgos de laboratorio también son inespecíficos, siendo la alteración hematológica más frecuente la anemia normocítica normocrómica ${ }^{1-3,7,8}$. De forma similar, nuestro paciente presentó importante pérdida de peso y anemia, así como rotura esplénica. La diarrea crónica puede estar asociada a enteritis isquémica por efecto de masa del tumor esplénico. Los hallazgos de imagen también son inespecíficos; se identifica con mayor frecuencia aumento de tamaño del bazo con áreas heterogéneas, y en algunos casos como lesiones nodulares solitarias ${ }^{9}$, por lo que no es infrecuente que la primera consideración diagnóstica sea un linfoma ${ }^{10,11}$. En nuestro caso no se pudieron evaluar los estudios de imagen prequirúrgicos, pues solo se contaba con el reporte escrito de tomografía y ultrasonido abdominales, que reportaban esplenomegalia con tejido esplénico de aspecto homogéneo, considerando inicialmente linfoma de la zona marginal esplénica.

Macroscópicamente el bazo suele estar aumentado de peso y de tamaño, hasta $3200 \mathrm{~g}$, aunque en algunos casos puede ser de dimensiones normales. La superficie externa es lisa y renitente, a menos que se presente con rotura, en la que se identifican soluciones de continuidad; en general, esta se presenta en bazos de gran tamaño (promedio de $1986 \mathrm{~g}$ ) y asociada a hemoperitoneo. Al corte, la superficie es heterogénea, en ocasiones se identifica una o múltiples lesiones nodulares, de bordes bien delimitados y de color blanco o rojizo, y en otros casos las lesiones son poco delimitadas, con áreas de necrosis, hemorragia o degeneración quística que pueden reemplazar por completo el órgano ${ }^{1,2}$. En nuestro caso, se presentó evidente rotura esplénica en un bazo con esplenomegalia masiva.

Microscópicamente la lesión es heterogénea, con una morfología variable en distintas áreas del tumor, por lo que es importante un extenso muestreo. En extendidos citológicos es posible identificar grupos de células fusiformes a pleomórficas, con núcleos ovales e hipercromáticos, en ocasiones con formación de papilas, pseudoacinos o pseudorosetas ${ }^{12}$. En los cortes definitivos, las áreas que son diagnósticas de angiosarcoma son las que demuestran formación de trayectos vasculares irregulares recubiertos por células endoteliales atípicas; sin embargo, las áreas con patrón de crecimiento sólido con áreas de morfología epitelioide o sarcomatoide son frecuentes, e incluso llegan a plantear un reto diagnóstico, ya que pueden simular carcinomas sarcomatoides, melanomas, mesoteliomas u otros sarcomas ${ }^{1,2,5,6}$. Por inmunohistoquímica se demuestra diferenciación vascular con positividad para CD31, CD34 y factor VIII principalmente, y en algunos casos reportados también pudo evidenciarse positividad para marcadores histiocíticos (CD68 y lizosima) $)^{1,11}$ y actina de músculo liso ${ }^{13}$. En nuestro caso, inicialmente se realizó impronta de la lesión con fines ilustrativos, en la que se evidenciaron grupos de células neoplásicas dispuestas en nidos conformados por células fusiformes, con citoplasma moderado, pleomórficas y cohesivas, lo que apoyó el diagnóstico de sarcoma, descartando procesos reactivos o linfoproliferativos. En los cortes definitivos se demostró un patrón clásico de angiosarcoma predominante $(80 \%)$, con áreas focales con patrón sólido (10\%) y papilar (10\%). Las reacciones de inmunohistoquímica confirmaron la naturaleza vascular de la lesión, excluyendo además otras posibilidades diagnósticas, como melanoma, leiomiosarcoma y carcinoma sarcomatoide, por la negatividad para HMB-45, calretinina y CKAE1/AE3. Algunos de los hallazgos relacionados con un comportamiento biológico agresivo de la neoplasia en nuestro caso fueron la presencia de extensa necrosis ( $90 \%$ de la lesión), pleomorfismo nuclear y elevado índice de proliferación por Ki-67 (>30\%), así como la presencia al momento del diagnóstico de metástasis hepáticas y pulmonares, diseminación ganglionar extensa y rotura esplénica.

La esplenectomía es el tratamiento de primera línea, aunque existen reportes en los que se ha utilizado radioterapia o quimioterapia como terapias 
adyuvantes, pero no han demostrado mejorar la supervivencia ${ }^{1,14,15}$.

En cuanto al pronóstico, por definición es malo. Hasta el $68 \%$ se presentan con enfermedad diseminada y el resto de los casos desarrollan metástasis en algún momento de la evolución, siendo los lugares afectados con mayor frecuencia el hígado y el pulmón, seguidos por los ganglios linfáticos, el hueso, los tejidos blandos, el tracto gastrointestinal y el cerebro. Sin embargo, el pronóstico es fatal en más del $90 \%$ de los casos al año del diagnóstico, independientemente de la diseminación y del tratamiento administrado ${ }^{1,2,7,8}$. Los patrones microscópicos presentes en la neoplasia y su proporción no tienen valor pronóstico. Los factores que se han asociado con un peor pronóstico son la rotura esplénica y el tamaño de la neoplasia, que reducen la expectativa de vida de 7 meses en promedio para los casos sin rotura esplénica a 3.9 meses para los casos con rotura, y de 9 meses para los casos con tumores menores de $5.0 \mathrm{~cm}$ a 5.7 meses para los casos con tumores mayores de $5 \mathrm{~cm}^{3,13}$.

Debido a los factores de mal pronóstico de nuestro paciente y a la ausencia de terapia adyuvante con repercusión en la supervivencia, solo se indicó tratamiento paliativo con seguimiento por consulta externa y fue dado de alta al presentar mejoría, pero el paciente no regresó.

\section{Conclusiones}

El angiosarcoma esplénico primario es una neoplasia poco frecuente que suele presentarse en etapas avanzadas, con un pronóstico sombrío, y puede ser causa de abdomen agudo secundario a rotura esplénica. En la actualidad no existe un tratamiento que mejore la supervivencia tras el diagnóstico, por lo que la esplenectomía sigue siendo el tratamiento de elección.

\section{Financiamiento}

Los autores declaran que no recibieron financiamiento de ninguna organización para la realización de este manuscrito.

\section{Conflicto de intereses}

Los autores declaran no tener ningún tipo de conflictos de intereses.

\section{Responsabilidades éticas}

Protección de personas y animales. Los autores declaran que para esta investigación no se han realizado experimentos en seres humanos ni en animales.

Confidencialidad de los datos. Los autores declaran que han seguido los protocolos de su centro de trabajo sobre la publicación de datos de pacientes.

Derecho a la privacidad y consentimiento informado. Los autores han obtenido el consentimiento informado de los pacientes y/o sujetos referidos en el artículo. Este documento obra en poder del autor de correspondencia.

\section{Bibliografía}

1. Neuhauser TS, Derringer GA, Thompson LD, Fanburg-Smith JC, Miettinen M, Saaristo A, et al. Splenic angiosarcoma: a clinicopathologic and immunophenotypic study of 28 cases. Mod Pathol. 2000;13:978-87.

2. Falk S, Krishnan J, Meis JM. Primary angiosarcoma of the spleen. A clinicopathologic study of 40 cases. Am J Surg Pathol. 1993;17:969-70.

3. Li R, Li M, Zhang LF, Liu XM, Hu TZ, Xia XJ, et al. Clinical characteristics and prognostic factors of primary splenic angiosarcoma: a retrospective clinical analysis from China. Cell Physiol Biochem. 2018;49:1959-69.

4. Langhans T. Pulsating cavernous neoplasm of the spleen with metastatic nodules to the liver. Vichows Arch Pathol Anat. 1879;75:273-91.

5. Hsu JT, Chen HM, Lin CY, Yeh CN, Hwang TL, Jan YY, et al. Primary angiosarcoma of the spleen. J Surg Oncol. 2005;92:312-6.

6. Hamid KS, Rodriguez JA, Lairmore TC. Primary splenic angiosarcoma. JSLS. 2010;14:431-5.

7. Verge J, Albiol J, Navas M, Martín C. Primary angiosarcoma of the spleen with liver metastases. Case report and literature review. Cir Esp. 2005;78:50-2.

8. Yang KF, Li Y, Wang DL, Yang JW, Wu SY, Xiao WD. Primary splenic angiosarcoma with liver metastasis: a case report and literature review. World J Gastroenterol. 2016;22:3506-10.

9. Kaza RK, Azar S, Al-Hawary MM, Francis IR. Primary and secondary neoplasms of the spleen. Cancer Imaging. 2010;10:173-82.

10. Suzuki K, Nakazato T, Mihara A, Sanada Y, Kakimoto T. Primary splenic angiosarcoma mimicking splenic lymphoma. Intern Med. 2010;49:203-4.

11. Chen X, Li H, Wang F, Liu H. Early detection and integral resection are keys to extend survival in patients suffered from primary angiosarcoma of the spleen: a care-compliant case report and literature review. Medicine (Baltimore). 2018;97:e9718.

12. Delacruz V, Jorda M, Gómez-Fernández C, Benedetto P, Ganjei P. Fine-needle aspiration diagnosis of angiosarcoma of the spleen: a case report and review of the literature. Arch Pathol Lab Med. 2005;129:1054-6.

13. Badiani R, Schaller G, Jain K, Swamy R, Gupta S. Angiosarcoma of the spleen presenting as spontaneous splenic rupture: a rare case report and review of the literature. Int J Surg Case Rep. 2013;4:765-7.

14. Abdallah RA, Abdou AG, Asaad NY, Al-Sharaky DR, Alhanafy AM. Primary epithelioid angiosarcoma of spleen; a case report and review of literature. J Clin Diagn Res. 2016;10:ED05-7.

15. Fiorentino MD, Monteiro JMC, Siqueira REB, Kim EIM, Curi AP, Ferrreira $C R$, et al. Primary splenic angiosarcoma: a rare entity often associated with rupture and hemoperitoneum. Autops Case Rep. 2019;9:e2019100. 